\title{
Fundamentación epistemológica del enfoque comunicativo en la enseñanza de lenguas extranjeras: una visión cognitivo-personalista
}

\author{
Carlos E. SEGADE ALONSO \\ Centro Universitario Villanueva \\ Departamento de Didácticas Aplicadas \\ csegade@villanueva.edu
}

Recibido: febrero 2011

Aceptado: marzo 2012

\begin{abstract}
RESUMEN
Los métodos de enseñanza de la lengua extranjera se fundamentan mayoritariamente en diferentes teorías psicológicas, lingüísticas o sociológicas, pero no es frecuente encontrar un enfoque o un recurso didáctico fundamentado antropológicamente. La antropología contemporánea, desde la óptica de la filosofía personalista, junto con la aportación de la lingüística cognitiva, hace posible la fundamentación epistemológica del acto de comunicación. La preferencia del enfoque comunicativo sobre otras técnicas depende más de su adecuación con la propia naturaleza de la acción comunicativa humana que de su eficacia implícita en relación al dominio de las competencias lingüísticas.

Palabras clave: epistemología, personalismo, lingüística cognitiva, enfoque comunicativo

Epistemological foundation of the communicative approach in second languages teaching: a cognitive-personal's point of view
\end{abstract}

\begin{abstract}
The methods of foreign language teaching are commonly grounded on various psychological, linguistic and sociological theories, but it is rare to find a particular didactic approach or resource which is anthropologically founded. Contemporary anthropology, from the Personalist Philosophy point of view, together with Cognitive Linguistics, enables the epistemological foundation of communication acts. A preference towards a communicative approach over and above other techniques depends more on its adjustment to the true nature of the human communicative action than on its implicit efficiency to reach a mastery of linguistic competences.
\end{abstract}

Key words: epistemology, personalism, cognitive linguistics, communicative approach 
Les bases épistémologiques de l'approche communicative dans l'enseignement des langues étrangères : une vision cognitive-personnaliste

\section{RÉSUMÉ}

Les méthodes d'enseignement des langues étrangères sont principalement fondées sur des théories psychologiques ou sociologiques, mais il est rare de trouver une approche ou d'une ressource pédagogique en fonction anthropologique. L'anthropologie contemporaine, du point de vue de la philosophie personnaliste, avec la contribution de la linguistique cognitive rend possible les fondements épistémologiques de l'acte de communication. La préférence de l'approche communicative á d'autres techniques dépend plus de la essence de l'action communicative humaine que son implicite efficacité en relation avec le domaine des compétences linguistiques.

Mots-clé: épistémologie, personnalisme, linguistique cognitive, approche communicative

SUMARIO: 1 . El problema del enfoque comunicativo y de la definición de persona; 1.1. $\mathrm{La}$ estructura de la persona; 1.2. Características de la acción; 1.3. Las relaciones interpersonales; 1.3.1. Las relaciones benevolentes; 1.3.2. Las relaciones de amistad; 1.3.3.El espacio conyugal; 1.3.4 Las relaciones malevolentes; 2. El lenguaje usado por la persona; 2.1 La pragmática de las relaciones interpersonales; 3 . Conclusiones: el acto de comunicación bajo la óptica cognitivo-personalista; 4. Referencias

\section{EL PROBLEMA DEL ENFOQUE COMUNICATIVO Y DE LA DEFINICIÓN DE PERSONA}

En la actualidad, el enfoque comunicativo en el aula es uno de los más apreciados por los docentes, bien sea por reacción a los modos tradicionales, más académicos, bien porque la edad de escolarización en dos idiomas haya bajado y se necesite una adecuación a los ciclos de enseñanza de educación infantil y primaria. Sea cual sea la razón por la que a los métodos comunicativos se les da más importancia, lo cierto es que los actos comunicativos cada vez ocupan un mayor espacio en los libros de texto ${ }^{1}$ y en las pruebas de competencias lingüísticas ${ }^{2}$.

Epistemológicamente, una metodología concreta es consecuencia de unas consideraciones previas de carácter muy variado, que pueden ir de la sociología a la psicología, pasando por la antropología (Madrid y Hughes, 2004, 38-67). Habitualmente, las técnicas o métodos didácticos en la enseñanza de la lengua extranjera han nacido como fruto de descubrimientos o presupuestos de la

\footnotetext{
${ }^{1}$ Se han revisado los libros destinados a alumnos de Educación Primaria de las editoriales Pearson-Longman, Oxford University Press y Cambridge University Press, del catálogo 2010, todos para lengua inglesa.

2 Véase, por ejemplo, el contenido de las pruebas conducentes a la obtención de la habilitación lingüística en la Comunidad de Madrid (Orden 1406/2006, de 14 marzo, en BOCM de 29 de marzo).
} 
psicología o de la lingüística y, por supuesto, de la adaptación de la propia experiencia de los profesionales de la enseñanza. Por ejemplo, el método audiolingüístico debe su fundamento teórico al conductivismo y al estructuralismo; directamente de las fuentes de la gramática generativo-transformacional, con su propuesta de transformaciones gramaticales, surgen los ejercicios de combinaciones de estructuras sintácticas, presentes incluso en los niveles más elementales de aprendizaje de las lenguas extranjeras.

Sin embargo, no es frecuente que los métodos se contrasten con los avances de la antropología y del conocimiento que las personas tenemos sobre nosotros mismos. No obstante, el progreso de la lingüística funcional y, sobre todo, de la lingüística cognitiva como uno de los paradigmas de aquella, nos lleva a interrogarnos sobre la posibilidad de someter a algunos de sus posicionamientos a la prueba de la razonabilidad antropológica, gracias a la predisposición que los lingüistas cognitivos tienen para contrastar sus hipótesis de trabajo con los datos aportados por otras disciplinas, especialmente la neurociencia. Aunque sea deseable, este contraste neurocientífico no será objeto de nuestro estudio, ya que previamente queremos someter a un análisis antropológico al acto de comunicación, como máximo exponente de una visión funcionalista del lenguaje.

La persona tiene una estructura compleja y es difícil de definir de forma breve, pero sí se puede definir por medio de los aspectos estáticos y dinámicos que componen dicha estructura (Burgos, 2008, 63-65). Entre estos últimos nos encontraremos la acción humana, donde ocupa un lugar preponderante el lenguaje $\mathrm{y}$, con los métodos propios del análisis antropológico, veremos si los principios que definen la acción coinciden con los requisitos exigibles a los actos de comunicación.

Esto nos lleva a plantear la hipótesis de que seguir un enfoque comunicativo en el aula es perfectamente complementario con una visión de la persona según los presupuestos de la filosofía personalista, si bien para ello hace falta partir paralelamente de los presupuestos de la teoría lingüística que sustenta dicho enfoque: la lingüística cognitiva. Por consiguiente, podría avanzarse que si admitimos estas hipótesis, primero, puede deducirse que cuanto más se adecuase una técnica a la naturaleza antropológica de la persona, mejor se aceptaría por parte del aprendiz de la lengua, ya que no supone aprender en un entorno artificial al que debe adaptarse antes de comenzar su proceso de aprendizaje, sino que, en segundo lugar, el docente podría adaptarse mejor a los intereses y al modo de aprendizaje del alumno cuanto mayor fuese el grado de personalización de la relación con el alumno, puesto que en todo momento se estaría en un entorno de comunicación real.

\subsection{La estructura de la persona}

La definición de persona, como hemos dicho, es muy compleja. Históricamente, por ejemplo, a la persona se le ha considerado sustancia (definición compartida con 
los objetos), ha sido conciencia o yo trascendental, etc., con la esperanza puesta en concentrar en una definición breve toda la complejidad que encierra el ser personal. La filosofía del primer tercio del siglo XX se adentró en el estudio del concepto bajo la perspectiva del entonces novedoso método fenomenológico, de forma que el ser humano pudiese aparecer ante los ojos del filósofo como una realidad sinfónica, susceptible de estudiarse desde varios puntos de vista.

Filósofos como Scheller, Stein, Marcel, Guardini, von Hildebrand, Mounier, Buber, Wojtyla, entre otros, han señalado la imposibilidad de encerrar en una sola definición, casi lexicográfica o al modo del que se definen los objetos, la multidimensionalidad de la persona. Brevemente, con el fin de ofrecer un panorama general de lo que venimos diciendo, podemos destacar someramente cuál sería esa multiplicidad de dimensiones (Burgos, 2008; Ferrer, 2002; Burgos, Cañas y Ferrer, 2006):

1.- Substancialidad: aunque la persona o sus circunstancias exteriores sufran cambios, la persona permanece siendo la misma.2.- Subjetividad: la persona se autoposee, tiene una gran riqueza interior que manifiesta al exterior mediante sus acciones.

3.- Dimensión corporal, temporal y espacial: la persona posee un cuerpo que le identifica y con el que es, que además vive inserto en un tiempo y en un espacio, aspectos ambos que condicionan su dimensión proyectiva.

4.- Apertura y trascendencia: la persona es un ser abierto, que sale de sí y se relaciona tanto con las cosas, como con otras personas y con Dios. Esta relación se produce mediante la acción.

5.- Identidad como varón o mujer: la persona es persona varón y persona mujer, aunque la identidad personal sea única e igual.

6.- Dignidad: todas las personas tienen dignidad en igualdad de condiciones con sus pares.

Todas estas dimensiones se plasman en una sola estructura: la persona. La definiríamos a partir de la suma de todas las dimensiones, si bien hay que tener en cuenta que estas no son partes escindibles del conjunto. Ninguna de las dimensiones restantes de la estructura sería posible sin el cuerpo, pero tampoco la afectividad o la libertad tienen sentido sin la inteligencia, y así sucesivamente. Hay una relación interdependiente entre ellas.

En la estructura personal hay aspectos de carácter estático y otros de carácter dinámico. Entre los primeros nos encontramos la corporeidad, la sensibilidad, la afectividad, la inteligencia, la libertad y la conciencia del yo personal. En el segundo grupo nos encontramos los aspectos de la estructura personal relacionados con "el otro", es decir, definidos por interrelación: la acción en general, el lenguaje, las relaciones interpersonales y el trabajo.

La persona interactúa con la realidad circundante. La persona actúa, o sea, lleva a cabo acciones que ponen de manifiesto la unidad de la persona. La acción personal es un dinamismo que la persona, como un 'yo', lleva a la existencia 
libremente con el objetivo de iniciar alguna transformación, pequeña o grande, del mundo circundante. Para evitar reduccionismos diremos que la persona no es acción, sino que la acción es de la persona. La persona vive en movimiento; la persona necesita actuar para vivir. La acción es causa con respecto a la realidad y necesaria con respecto a la persona.

Ya que la persona transforma la realidad, habrá que estudiarla también en su dimensión transformadora, o sea, en la praxis, a la que pertenece el lenguaje. El lenguaje es el instrumento en virtud del cual la persona interactúa con otras personas que, a su vez, transformarán su realidad circundante.

\subsection{Características de la acción}

La estructura de la acción humana es bidimensional (Burgos, 2009, 100): transitiva e intransitiva simultáneamente.

Es transitiva en cuanto que el sujeto (la persona que lleva a cabo la acción) hace algo (llámese trabajo, actividad de ocio o cualesquiera otras actividades) sobre un objeto distinto de sí mismo. La acción no es la persona, pero la persona crea la acción y 'deja hecho' un producto objetivo de su acción.

Al mismo tiempo, mediante la acción, la persona se modifica a sí misma. Tras la experiencia de la acción, la persona lleva a cabo un aprendizaje, refuerza su autoposesión, causa la transformación, en algún grado, del mundo circundante, etc., es decir, ha interactuado con el mundo y al mismo tiempo su experiencia vital ha aumentado.

Aunque la acción se haya hecho solo con voluntad transitiva, lo cierto es que la persona, tras ver las consecuencias de su acción, aprende algo nuevo, conoce una nueva realidad, la valora, la juzga, o adquiere un nuevo conocimiento. De cualquier forma, lo que hace es sufrir una transformación interior: tras una acción hay una modificación de la persona en virtud de los efectos de esa acción. Esto es lo que llamamos intransitividad de la acción personal.

Las transformaciones de la realidad o de los objetos son las más evidentes para los sentidos, por lo que podemos afirmar que también lo es la dimensión transitiva de la praxis personal. Se manifiestan en los avances científicos y técnicos y las reflexiones metacognitivas.

La dimensión intransitiva a veces es menos tangible, pero no por eso menos evidente. La admiración, el asombro, el entusiasmo, la fascinación, la cultura, son el resultado de la dimensión intransitiva: todas ellas incrementan en cualidad la existencia personal tras valorar contemplativamente los resultados o los productos de la acción.

La persona, por consiguiente, modela su existencia por interacción, bien con las cosas, objetos de la realidad, bien con otras personas. El hecho de que su transformación interna se produzca en el intercambio de acciones abre lo que consideramos como un espacio metafísico trascendente, crucial para entender la idea de persona. La acción no es solo un movimiento de un sujeto válido por sí 
mismo, sino que se realiza abierto al otro cuando no exclusivamente dirigido al otro. De aquí que podamos decir que la naturaleza del hombre es proyectiva (Marías, 1987) en tanto que la biografía personal requiere un proyecto de vida que fácilmente puede compartirse con otra $\mathrm{u}$ otras personas, o al que la persona voluntariamente se suma.

En este amplio campo para la acción, o sea, la relación con el otro, la persona obtiene su máximo desarrollo, ya que al menos cuatro de las dimensiones de la persona (afectividad, espiritualidad, corporalidad y psiquismo) tienen su realización plena en la acción trascendente.

\subsection{Las relaciones interpersonales}

Lo que distingue la filosofía centrada en la persona de los planteamientos idealistas de los siglos XIX y XX es, precisamente, la relevancia que se da a la relación con las otras personas, como se ha podido poner de manifiesto en los párrafos anteriores. Supone un esfuerzo de interiorización, que no de subjetivismo, lo que equivale a ponerse en el papel del sujeto-persona y tratar de averiguar qué ve a través de sus ojos y qué capta a través de su mente; se trata de contemplar todos los aspectos que le afectan, mirando como mira una persona la realidad circundante. Dentro de esa realidad están también las otras personas. Más aún, la realidad no se entendería sin las demás personas.

Las relaciones interpersonales son un encuentro entre un Yo y un Tú, basado en la reciprocidad (Buber, 2005, 15). La persona se encuentra con otras personas a lo largo de su existencia, pero según de qué persona se trate, así va a ser el modo de establecer una relación con ella. Por eso, según sea el encuentro, así se modelará la relación que una persona mantiene con las demás. Esto tiene una importancia vital, ya que supone por parte de la persona la activación de sus competencias socioculturales.

Aunque siempre resulta difícil categorizar los comportamientos, podemos establecer tipos de relaciones generales según sean los modos de relación y los contextos peculiares que rodean a las personas. Los tipos de relaciones son los siguientes: benevolentes, amistosas, conyugales y malevolentes.

\subsubsection{Relaciones benevolentes}

Bajo este epígrafe, en un nivel más elemental, se encuentran las relaciones que llamamos instrumentales. En este tipo de relación necesitamos a otra persona para conseguir algo, por ejemplo, la relación con la cajera del supermercado donde compramos el pan o con el camarero que nos sirve el café de media mañana. Las actuaciones entre ambos están gobernadas estrictamente por las reglas de urbanidad.

En un nivel siguiente nos encontramos las relaciones de solidaridad, más cordiales que las anteriores, ya que podemos llegar a compartir un conocimiento algo "más personal". Por ejemplo, la relación que se mantiene con los compañeros 
de trabajo. Se mantiene una relación meramente circunstancial, que durará mientras dure la relación laboral, que establece una relación personal en aras de un proyecto común.

\subsubsection{Las relaciones de amistad}

La amistad es un modo de relación recíproca no instrumentalizada donde no hay más objetivo que compartir momentos concretos de un proyecto vital, manteniendo la independencia en la mayoría de las acciones.

La amistad intersexual puede derivar en el amor, que se sublima cuando se focaliza en una o en varias personas concretas, de tal forma que las afirmaciones "te quiero" o "te amo" lingüísticamente expresadas tienen pleno sentido.

\subsubsection{El espacio conyugal}

El ámbito natural en el que desemboca el enamoramiento es el espacio trascendente conyugal (Segade, 2007). Este espacio es el creado por ambos cónyuges para desarrollar juntos su proyecto vital.

\subsubsection{Las relaciones malevolentes}

La antropología no suele estudiar la acción humana desde un punto de vista negativo. Es decir, se supone que las relaciones humanas normalmente son benevolentes, con clasificaciones más o menos similares a la que hemos seguido. Sin embargo, odiar es también una relación personal. Se basa en romper voluntariamente cualquier tipo de relación benevolente con la otra persona hasta incluso desear su desaparición física, porque el odio se proyecta sobre la persona entera, aunque el objeto del odio sea una o varias características concretas de la otra persona. En definitiva, es el rechazo absoluto a establecer ninguna relación con el otro.

En todos estos tipos de relaciones la persona verbaliza sus intenciones con recursos lingüísticos. La persona selecciona el registro que utilizará al igual que la función del lenguaje que activará (por ejemplo, la manipulativa para las relaciones benevolentes instrumentales). Pero esas subcompetencias lingüísticas que se activan se hacen según un contexto y un conocimiento de la totalidad de la realidad en la que se está moviendo la persona para decidir en qué tipo de relación debe establecer su discurso. Esto quiere decir que la elección del léxico y recursos pragmáticos está socioculturalmente motivada.

\section{EL LENGUAJE USADO POR LA PERSONA}

Como hemos visto en los párrafos anteriores, la persona se mueve y vive en la interacción con los objetos y las personas. La relación con las cosas es necesaria (cubre tanto las necesidades más fundamentales como la satisfacción de deseos de carácter superfluo) mediante la transformación, alteración y manufactura de las cosas. La estructura antropológica de la persona también prevé la interacción con 
las demás personas en distintos niveles de relación, lo que nos habla de un contexto, una intencionalidad y un deseo personal de comunicación. Con todo esto podemos avanzar que el lenguaje es antropológicamente una acción y, semánticamente, fundamentado en una experiencia.

El lenguaje humano es también objeto de disputa en cuanto a la descripción de su naturaleza. Aunque más o menos existe un consenso en cuanto a su constitución estructural, es cierto que este aspecto no es el más sobresaliente, a pesar de que ese sea prácticamente el único objeto de estudio de los lingüistas de tradición generativista o distribucionalista (Mairal Usón, Peña Cervel, Cortés Rodríguez y Ruiz de Mendoza Ibáñez, 2010, 292). Incluso terminológicamente, a veces se aprecian confusiones por la aplicación de los mismos términos a realidades distintas. Mientras que para la tradición chomskyana competencia gramatical es la facultad de los hablantes de producir expresiones lingüísticas, desde la perspectiva funcional la competencia comunicativa añade a la anterior la capacidad de adaptación del hablante a las convenciones de la comunidad lingüística (Mairal Usón, Peña Cervel, Cortés Rodríguez y Ruiz de Mendoza Ibáñez, 2010, 296).

El cognitivismo explica que el significado se fundamenta en el procesamiento racional de la experiencia corporal, con toda la complejidad que esto implica. Esto se opone a la concepción que el objetivismo (generativismo) tiene del pensamiento y del procesamiento de la información. A continuación exponemos de forma esquemática lo que Cuenca y Hilferty $(2007,16)$ recogen sobre los fundamentos de ambos enfoques y que resumimos para ilustrar nuestra hipótesis:

Concepción objetivista del pensamiento humano:

- Pensar es manipular símbolos abstractos, que se relacionan directamente con el mundo.

- El pensamiento es independiente del cuerpo humano.

- El pensamiento es atomístico.

- El pensamiento es lógico y puede ser formalizado y descrito a partir de valores de verdad.

Concepción experiencialista:

- El pensamiento responde a una estructura ecológica, en el sentido de que el pensamiento es algo más que la mera manipulación de símbolos abstractos, sino que tiene que ver con una estructuración conceptual global del mundo.

- El pensamiento tiene carácter corpóreo, es decir, se basa en la experiencia corporal humana. El embodiment o corporeización pone de manifiesto que el pensamiento surge de la experiencia corpórea total. 
- El pensamiento tiene propiedades gestálticas ${ }^{3}$, o sea, que la combinación de las partes del lenguaje forma unidades simbólicas que son más simples que la suma de sus partes, por consiguiente, se opone al atomismo objetivista en virtud del cual el lenguaje es la combinación de unidades complejas, analizables en otras simples de igual rango.

- El pensamiento es imaginativo y solo puede describirse por modelos cognitivos. El experiencialismo supera la lógica proposicional mediante la explicación del lenguaje en función de modelos cognitivos, ya que la verdad de una proposición no es definible como lo puede ser la matemática, aplicando lógica matemática, sino que responde a modelos conceptuales abstractos complejos, mayormente analógicos, metafóricos y metonímicos.

Estos principios fundamentan a su vez los principios de la lingüística cognitiva (Cuenca y Hilferty, 2007, 19; Langacker, 2008, 3-89), que también resumimos en estos puntos:

- El lenguaje no puede separarse del enfoque comunicativo, por tanto es necesario el lenguaje en su uso real.

- El pensamiento se organiza mediante procesos de categorización, es decir, de estructuras conceptuales, relaciones prototípicas y de semejanza de familia con límites difusos, no discretos.

- El lenguaje es eminentemente simbólico, no se puede separar la semántica del análisis formal.

- La gramática es el resultado, no el origen, de la estructuración y simbolización del contenido semántico de una forma fonológica.

- La caracterización del lenguaje es dinámica y la gramática está en continua evolución.

Si analizamos con atención estos presupuestos, se deduce que desde el cognitivismo se comprende el lenguaje como una de las posibles soluciones que tiene la persona ante una necesidad de tipo comunicativo (Mairal Usón, Peña Cervel, Cortés Rodríguez y Ruiz de Mendoza Ibáñez, 2010, 294). La persona es algo más que un ser animado con capacidad lingüística que instintivamente pone en funcionamiento un órgano lingüístico ante un estímulo exterior. La persona activa intencionalmente todos los recursos posibles y todas sus facultades orgánicas para que su intención de comunicación tenga éxito. En un acto comunicativo se activa la facultad lingüística, puramente orgánica, de los órganos fonadores y de las áreas cerebrales involucradas, lo que trae consigo que se pongan en marcha la capacidad epistémica, la capacidad lógica, la capacidad perceptiva y la capacidad social.

\footnotetext{
${ }^{3}$ Gestalt: "a whole that we human beings find more basic than the parts" (Lakoff and Johnson, 2003, 70).
} 


\subsection{La pragmática de las relaciones interpersonales}

Las relaciones interpersonales, sean del tipo que sean, nacen de una intencionalidad, lo que en filosofía del lenguaje se viene denominando fuerza ilocutiva. Según la teoría de los actos de habla, es bien conocido que el discurso puede describir la realidad o modificarla, de acuerdo con la intención del emisor. Aun siendo esto cierto, lo que primero se produce en el hablante es la adaptación de su futuro discurso al contexto en el que está. Tanto la lingüística cognitiva (Lakoff y Johnson, 2003, 3-40; Johnson, 2008, 1-15) como la teoría de los actos de habla (Searle, 1998, 135-161) y la psicología cognitiva (Belinchón, Igoa y Rivière, 2009), esta última desde la noción de deixis, dotan al contexto de una importancia primordial a la hora de que (al menos) dos personas establezcan un acto de comunicación.

El conocimiento del mundo que tiene la persona es enciclopédico (Lakoff y Johnson, 2003, 77-86) y es la suma del puro raciocinio y de la experiencia física de la realidad. La persona es una, cuerpo y mente, razón y corporeidad, por tanto, la experiencia corporal colabora en el conocimiento del mundo al igual que la capacidad de raciocinio. Como afirman Belinchón, Igoa y Riviére $(2009,680)$ la capacidad de interpretación de la realidad personal se detecta a edades extremadamente tempranas, por ejemplo, en los neonatos ante la cara y voz humanas o la sincronización de la actividad motora de los bebés con los ciclos habla/silencio de la madre desde las primeras horas de vida.

El conocimiento enciclopédico de la realidad lleva pareja la capacidad humana de relacionar realidades similares para hacerse entender; este es el origen de la metáfora, en virtud de la cual una realidad se verbaliza con el lenguaje propio de otra realidad. Lógicamente, los participantes en el acto de comunicación deben conocer ambas realidades para poder hacer la traslación de la una a la otra. Pero las realidades se conocen en función de los parámetros culturales en los que se está inmerso. Por tanto, las metáforas son culturalmente motivadas. El lenguaje es primordialmente metafórico (Lakoff y Johnson, 2003, 3-6), por lo que lógicamente, el lenguaje está culturalmente motivado en sus estructuras y en su significado.

El proceso de aprendizaje de las metáforas es lento y supone un dominio excelente de la lengua extranjera. Téngase en cuenta que el proceso de aprendizaje de metáforas y de funciones del lenguaje es lento incluso en la lengua materna. Como muestran Filippova y Astington (2010) los niños de edades comprendidas entre cinco y nueve años tienen serias dificultades para comprender un discurso irónico en alguna de sus variantes.

La razón de esta dificultad estriba en que las personas crean constructos cognitivos idealizados (Mairal Usón, Peña Cervel, Cortés Rodríguez y Ruiz de Mendoza Ibáñez, 2010, 234), es decir, estructuras cognitivas que tienen como finalidad representar la realidad desde una perspectiva concreta, lo que Lakoff bautizó como Modelo Cognitivo Idealizado (en adelante MCI), y que son 
entramados de conceptos realmente complejos y muy costosos de adquirir, incluso en la lengua materna. Los MCIs son categorías abiertas que están compuestas de relaciones de entidades con un grado de mayor o menor prototipicidad. En Mairal Usón, Peña Cervel, Cortés Rodríguez y Ruiz de Mendoza Ibáñez (2010, 235-7) se pueden encontrar ejemplos de Fillmore (los sentidos de la palabra 'bachelor') o Lakoff (la palabra 'mother') en los que se detecta un consenso sobre su significado prototípico pero que es difícilmente aplicable a tipos concretos de esa serie (por ejemplo, como sostiene Fillmore, nadie definiría a un soltero poniendo como modelo a Tarzán o al Papa, ya que en ambos casos existen características peculiares que son prioritarias, aunque en ambos casos también se les pueda caracterizar con el rasgo de 'solteros'). Por eso, en el aprendizaje de una lengua no basta con la traducción directa de una voz, sino que también es necesario ir dando a entender todas las posibles combinaciones de esa palabra en diferentes contextos, algunos de ellos equivalentes en varias lenguas y otros totalmente exclusivos de una sola.

La experiencia, como veíamos, comienza en los primeros momentos de vida y por tanto es exclusivamente personal y, como la persona misma, incomunicable. El lenguaje intenta suplir esa imposibilidad antropológica mediante la expresión de las experiencias personales de la manera más exacta posible por medio del lenguaje metafórico y metonímico, y de todos los recursos de la pragmática. Pero lo más importante en el nivel de estudio que estamos llevando a cabo es que, en un acto de comunicación, la persona activa su capacidad subjetiva de interpretar el mundo que le rodea, adaptando su discurso y todas sus capacidades comunicativas a su intención de comunicarse.

La visión cognitiva es complementaria con la concepción antropológica personalista en tanto que, como se vio con anterioridad, la persona debe adaptarse al contexto de las relaciones personales de forma natural con sus propios recursos. Una persona puede pasar por una situación que requiera un registro propio de las relaciones meramente benevolentes, pero que no deben confundirse entre ellas o con las de amistad o las conyugales. El conocimiento de los registros de unas relaciones y otras son el fundamento básico de cualquier educación cívica, aún en la lengua materna. Paralelamente, es necesario que el estudiante de una segunda lengua sea consciente de los distintos registros de output que están a su disposición y que debe administrar. Muy frecuentemente, además, hay que advertir al aprendiz que hay ciertas expresiones comunes en la cultura propia, sean pragmáticas o meramente lingüísticas, que se interpretan como propias de relaciones malevolentes en otras culturas. Con este propósito no solo el alumno sino también el docente debe ser consciente del reto que supone abrir un espacio intercultural (Vez y González Piñeiro, 2004). 


\section{CONCLUSIONES: EL ACTO DE COMUNICACIÓN BAJO LA ÓPTICA COGNITIVO-PERSONALISTA}

El enfoque comunicativo en la enseñanza de la lengua extranjera tiene ya un amplio recorrido. En un sentido amplio y poco académico, erróneamente se etiqueta como comunicativo a cualquier método que obligue a seguir unas pautas de simulación de comunicación oral o escrita, porque se asocia típicamente la función comunicativa con la capacidad de producir un output lingüístico o de exponerse a un input fonético o escrito y comprenderlo. De hecho, esta apreciación es un lugar común entre los estudiantes de didáctica de las lenguas, adjudicando la categoría de comunicativo, por ejemplo, al método audiolingüístico, de tal forma que se confunde éste con el enfoque oral o situacional.

En un sentido más estricto, los enfoques oral y situacional buscan la inferencia (por parte del aprendiz) de estructuras concebidas bajo el paraguas común del estudio de la gramática. El aprendizaje limitado a la comprensión de los fenómenos lingüísticos, los propios de una lengua junto con los comunes a todas las lenguas, es una propuesta de adquisición de una segunda lengua más propia de los defensores de la existencia de una gramática universal (Cook y Newson, 2003).

El enfoque funcional, a pesar de sus varios representantes y visiones particulares, fundamenta el lenguaje en el significado y, por consiguiente, en la comunicación, de manera que la gramática pasa a ser un inventario de unidades simbólicas estructuradas (Cuenca y Hilferty, 2007, 187; Langacker, 2008; Mairal Usón, Peña Cervel, Cortés Rodríguez, y Ruiz de Mendoza Ibáñez, 2010), de naturaleza semántica, que forman un continuum con la morfología y la sintaxis. Una metodología basada en un enfoque comunicativo incide en las funciones del lenguaje antes que en el vocabulario o la gramática (Megías y Santamaría, 2004, 319; Larsen-Freeman, 2003, 121-136). En los modelos no funcionales la necesidad de formalización lingüística (diseño de una gramática) crea modelos idealizados basados en la prototipicidad de sus componentes, de tal manera que se descartan como excepcionales los usos que no entran en los márgenes de la categorización, aunque sean reales. Básicamente este es el fundamento que permite la crítica de técnicas didácticas que, como el método audiolingüístico, aplica estructuras gramaticales a situaciones prototípicas que no pueden prever la contingencia inherente a cualquier interacción humana, al igual que sucede con las limitaciones semánticas que impone un aprendizaje basado en transformaciones estructurales.

Como punto alrededor del cual gira todo el enfoque comunicativo se encuentra la creación de situaciones que se definen como actos de comunicación, o sea, todas las tareas, actividades, juegos o recursos didácticos (preferentemente orales, pero no necesariamente) que compartan estas tres características (Larsen-Freeman, 2003, 129):

1) hay necesidad de comunicación real de información nueva para al menos uno de los actores de la acción comunicativa (information gap);

2) hay opciones reales de cómo y qué se puede decir de una cosa (choice); 
3) hay una necesidad de verificación por parte de los actores de que la comunicación ha sido positiva (feedback), consecuentemente, a través del propio desarrollo coherente de la comunicación se valora si se ha alcanzado el propósito originario por el que se inició.

En términos antropológicos, la persona aprende activando su capacidad de relación con el otro, por lo tanto, mantiene una relación transitiva cuando menos de carácter benevolente cuyo resultado produce un aprendizaje y reafirma, asimismo, el principio de intransitividad de cualquier acción humana.

En un acto de comunicación, el lenguaje se usa de forma contextualizada y la interrelación puede conllevar la negociación del significado, lo cual quiere decir que el estudiante pone en relación el output fonético con una multiplicidad de opciones conceptuales que ofrece el contexto y el registro cultural, de tal manera que de la unión entre ambos surja el significado. De entre las muchas acepciones que puede tener una palabra, el estudiante selecciona cuál es la que más concuerda con el contexto. De esta forma, incluso en ámbitos académicos formales, se pueden dar condiciones similares a las de una situación de adquisición de una lengua en un entorno natural monolingüe.

El acto de comunicación fuerza al estudiante a conocer los usos pragmáticos de la lengua, de manera que en la repetición de actos de comunicación se pueden cubrir las competencias lingüísticas, sociolingüísticas y pragmáticas exigibles por el Marco Común Europeo (Vez y González Piñeiro, 2004).

Según las bases propuestas por el Marco Común Europeo que se recomienda seguir a los profesores de lenguas (Little y Perclová, s.f.) el estudiante debe dominar las siguientes subcompetencias: marcadores de relaciones sociales; fórmulas de cortesía; diferencias de registro; expresiones de sabiduría popular; dialectos y acentos. Las tres primeras pueden fácilmente practicarse mediante actos de comunicación enmarcados en los tipos de interrelaciones de las que hemos venido hablando, incluyendo refranes, frases hechas y frases proverbiales. Solo los dialectos y acentos quedarían fuera de esos actos de comunicación en un aula, al menos que se disponga de profesorado nativo de diferentes orígenes o material muy variado (grabaciones ad hoc o escogidas de medios de comunicación) sobre los que pudieran hacerse tareas que impliquen posteriores actos de comunicación.

Entre las subcompetencias pragmáticas que el Marco Común prevé nos encontramos las relacionadas con el discurso (contexto, cohesión y coherencia) y las funcionales (función ideativa, manipulativa, heurística e imaginativa) que, al igual que las socioculturales, pueden ir subsumidas en la intencionalidad de un acto de comunicación, ya que la dimensión transitiva de la acción lingüística exige la funcionalidad por ser transformadora, en algún modo, de la realidad.

Esto es por lo que podemos afirmar que el enfoque comunicativo en la enseñanza de la lengua extranjera tiene dos fundamentos epistemológicos: la antropología de la acción personal y la lingüística cognitiva. La primera en cuanto a la relación educativa de los actores que intervienen en la comunicación y la 
segunda en cuanto a la forma, influencias y funciones del discurso empleado en la acción de comunicación.

La antropología contemporánea nos informa de que la acción humana es transitiva e intransitiva. La acción, amalgama de transformación de la realidad, comportamiento ético y aprendizaje, se produce por interrelación en un contexto culturalmente motivado, de forma que la persona debe adaptarse a él por medio del vehículo de adaptación por excelencia: el lenguaje humano.

La persona que utiliza el lenguaje lo usa primordialmente para establecer alguno de los tipos de relaciones personales posibles. Como esas relaciones están contextualizadas, el lenguaje también debe estarlo, activando así las competencias socioculturales y pragmáticas de las que dispone.

La práctica repetida de actos de comunicación reales, sin minusvalorar la utilización de otros recursos didácticos, reforzaría no solo el conjunto de competencias lingüísticas (subcompetencias léxicas, gramaticales, semánticas, fonológicas, ortográficas y ortoépicas), sino también el resto de las que hemos señalado, impidiendo que el alumno se vea incapacitado de interpretar el mundo en la segunda lengua, con toda su riqueza metafórica (pragmática).

\section{REFERENCIAS BIBLIOGRÁFICAS}

BELINCHÓN, M., IGOA, J.M. y RIVIÉRE, A. (2009): Psicología del lenguaje. Investigación y teoría, Madrid, Trotta.

BUBER, M. (2005): Yo y Tú, Madrid, Caparrós Editores.

BURGOS, J.M. (2008): Antropología: una guía para la existencia, Madrid, Palabra.

BURGOS, J.M. (2009): Reconstruir la persona, Madrid, Palabra.

BURGOS, J.M. (Coord.).(2007): La filosofía personalista de Karol Wojtyla, Madrid, Palabra.

BURGOS, J.M., CAÑAS, J.L., FERRER, U. (Coords.)(2006): Hacia una definición de la filosofia personalista, Madrid, Palabra.

COOK, V.J. y NEWSON, M. (2003): Chomsky's Universal Grammar, Oxford, Blackwell.

CUENCA, M.J. y HILFERTY, J. (2007): Introducción a la lingüistica cognitiva, Barcelona, Ariel.

FERRER, U. (2002): ¿Qué significa ser persona?, Madrid, Palabra.

FILIPOVA, E. y ASTINGTON, J.W. (2010): Children's Understanding of SocialCognitive and Social-Communicative Aspects of Discourse Irony. Child Development, 81(3), 913-928.

JOHNSON, M. (2008): The Meaning of the Body. Aesthetics of Human Understanding, Chicago, The University of Chicago Press.

LAKOFF, G. y JOHNSON, M. (2003): Metaphors we Live by, Chicago, The University of Chicago Press. 
LANGACKER, R.W. (2008): Cognitive Grammar: a basic introduction, Oxford, OUP.

LARSEN-FREEMAN, D. (2003): Techniques and Principles in Language Teaching, Oxford, OUP.

LEVINSON, S.C. (2008): Pragmatics, Cambridge, CUP.

LITTLE, D. y PERCLOVÁ, R. (s.f.): The European Language Portfolio: a guide for teachers and teacher trainers. disponible en http://www.coe.int/T/DG4/Portfolio/documents [consulta: 10 de septiembre de 2010]

MARÍAS, J. (1987): Antropología metafísica, Madrid, Alianza.

MADRID, D. y HUGHES, S.P. (2004): Epistemology of foreign language didactics. En TEFL in Primary Education, MADRID, D. y McLAREN (Coords.), Granada, Universidad de Granada, 38-71.

MADRID, D. y McLAREN, N. (Coords.)(2004), TEFL in Primary Education, Granada, Universidad de Granada.

MAIRAL USÓN, R., PEÑA CERVEL, M.S., CORTÉS RODRÍGUEZ, F.J. y RUIZ DE MENDOZA IBÁÑEZ, F.J. (2010): Teoría lingüistica. Métodos, herramientas y paradigmas, Madrid, Editorial Universitaria Ramón ArecesUNED.

MEGÍAS, M. y SANTAMARÍA, C. (2004): Sociolinguistic and pragmatic competences. En TEFL in Primary Education, MADRID, D. y McLAREN (Coords.), Granada, Universidad de Granada, 315-340.

PÉREZ VALVERDE, C. y MUROS, J. (2004): Discourse competence in the EFL classroom. En TEFL in Primary Education, MADRID, D. y McLAREN (Coords.), Granada, Universidad de Granada, 385-408.

SEARLE, J.R. (1998): Mind, Language and Society. Philosophy in the Real World, New York, Basic Books.

SEGADE, C. (2007): El amor conyugal como espacio trascendente. En La filosofía personalista de Karol Wojtyla, Burgos, J.M. (Ed.) ,Madrid, Palabra, 107-114.

TEJADA MOLINA, G. y PÉREZ CAÑADO, M.L. (2004): Oral communication and interaction. En TEFL in Primary Education, MADRID, D. y McLAREN (Coords.), Granada, Universidad de Granada, 178-217.

VEZ, J.M. (2000), Fundamentos lingüisticos en la enseñanza de lenguas extranjeras, Barcelona, Ariel.

VEZ, J.M. y GONZÁLEZ PIÑEIRO, M. (2004): Intercultural competence and the European dimensión. En TEFL in Primary Education, MADRID, D. y McLAREN (Coords.), Granada, Universidad de Granada, 342-383. 